\title{
Imaging features of 2019 novel coronavirus pneumonia
}

\section{$\mathrm{Xi} \mathrm{Xu^{1 }}$ - Chengcheng $\mathrm{Yu}^{2} \cdot$ Lieguang Zhang ${ }^{2} \cdot$ Liangping Luo ${ }^{1} \cdot$ Jinxin Liu $^{2}$}

Published online: 14 February 2020

(C) Springer-Verlag GmbH Germany, part of Springer Nature 2020

Dear Sir,

Recently, a type of pneumonia caused by a novel Betacoronavirus, the 2019 novel coronavirus (2019-nCoV), has spread in China, mainly in Wuhan [1]. It is known that cases of this disease have also been identified in other provinces of China [2].

A 53-year-old man has lived in Wuhan for many years, and recently came to Guangzhou, China. He was admitted to the

Please note that due to the time sensitive nature of the work presented in this article, standard peer-review has been bypassed to ensure rapid publication. The article has been directly assessed by the Editor-in-Chief.

This article is part of the Topical Collection on The Infection and Inflammation

Liangping Luo

tluolp@jnu.edu.cn

Jinxin Liu

Liujx83710378@126.com

$\mathrm{Xi} \mathrm{Xu}$

736461654@qq.com

Chengcheng $\mathrm{Yu}$

1515185140@qq.com

Lieguang Zhang

zhlieguang@126.com

1 Department of Medical Imaging Center, the First Affiliated Hospital, Jinan University, No. 613, Huangpu Road West, Tianhe District, Guangzhou 510630, China

2 Department of Radiology, Guangzhou Eighth People's Hospital, Guangzhou Medical University, No. 8, Huaying Road, Baiyun District, Guangzhou 510060, Guangdong Province, China hospital with fever and cough in the last 2 days, and his highest body temperature was $38.3{ }^{\circ} \mathrm{C}$ before admission. The laboratory examinations showed decreased white blood cell count $\left(2.50 \times 10^{9} / \mathrm{L}\right)$ and elevated C-reactive protein $(16.2 \mathrm{mg} / \mathrm{L}$; normal range $0-10 \mathrm{mg} / \mathrm{L})$.

Non-contrast-enhanced chest computed tomography (CT) (Fig. 1a, b) showed multiple, bilateral, peripheral, patchy ground glass opacities, lobular, and subsegmental, with fading margins. The lesions had mainly a subpleural distribution.

CT also demonstrated slight thickening of the adjacent pleura (yellow arrow) and interlobular septa in the lesion (red arrow). Subsequently, the patients had laboratoryconfirmed 2019-nCoV infection by real-time polymerase chain reaction (PCR). After 5 days, CT scan (Fig. 1c, d) showed enlarged lesions, compared with previous images, and progressive ground glass opacities in both lower lobes, indicating disease progression.

Infection with 2019-nCoV was confirmed based on the patient's epidemiological history, clinical manifestations, imaging characteristics, and laboratory tests.

Bilateral, multiple, patchy, or ground glass opacities, with subpleural distribution, were the lung CT appearances of this patient, affected by 2019-nCoV pneumonia. These characteristics should be recognized by professionals working in imaging departments. 
Fig. 1 CT scan of the patient. CT demonstrated slight thickening of the adjacent pleura (yellow arrow) and interlobular septa in the lesion (red arrow)
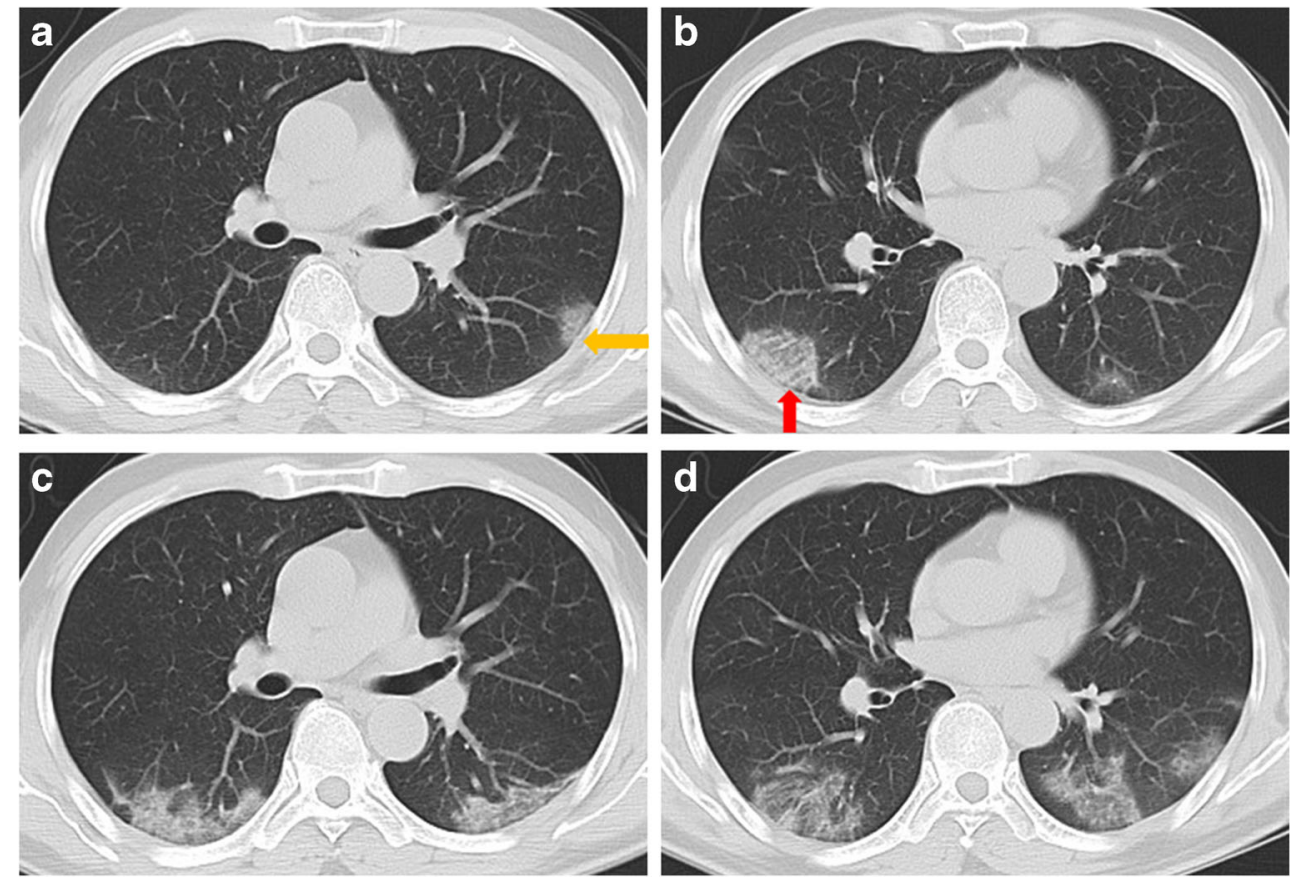

Authors' contributions JL and LL designed the study. XX was a major contributor in writing the manuscript. LZ and CY revised it critically for important intellectual content. All authors have read and approved the manuscript.

\section{Compliance with ethical standards}

Conflict of interest The authors declare that they have no conflicts of interest.

Ethics approval Patient's consent for publication of fully anonymized images was waived.

\section{References}

1. Zhu N, Zhang D, Wang W, Li X, Yang B, Song J, et al. A novel coronavirus from patients with pneumonia in China, 2019 [J]. N Engl J Med. 2020. https://doi.org/10.1056/NEJMoa2001017.

2. Huang C, Wang Y, Li X, Ren L, Zhao J, Hu Y, et al. Clinical features of patients infected with 2019 novel coronavirus in Wuhan, China [J]. Lancet. 2020. https://doi.org/10.1016/S0140-6736(20)30183-5.

Publisher's note Springer Nature remains neutral with regard to jurisdictional claims in published maps and institutional affiliations. 